\title{
LINC00857 promotes cell proliferation and migration in colorectal cancer by interacting with YTHDC1 and stabilizing SLC7A5
}

\author{
SHU TANG ${ }^{1}$, QI LIU ${ }^{2}$ and MING XU ${ }^{3}$ \\ ${ }^{1}$ Department of Internal Medicine-Oncology; ${ }^{2}$ Hospital Medical Department; ${ }^{3}$ Department of Gastrointestinal Surgery, \\ Chenzhou No. 1 People's Hospital, Chenzhou, Hunan 423000, P.R. China
}

Received December 21, 2020; Accepted March 12, 2021

DOI: $10.3892 / \mathrm{ol} .2021 .12839$

\begin{abstract}
Colorectal cancer (CRC) is one of the most lethal malignances in humans. Hence, it is of great significance to identify regulatory molecules in CRC progression. Accumulating evidence has demonstrated that long non-coding RNAs (lncRNAs) are involved in cancer malignancy. It has been reported that long intergenic non-protein coding RNA 857 (LINC00857) acts as a vital oncogene in many types of cancer by promoting cell proliferation and migration. However, the role of LINC00857 in CRC remains unclear. In the present study, LINC00857 was upregulated in CRC tissue samples and cells. Next, in vitro loss-of-function experiments demonstrated that LINC00857 knockdown suppressed CRC cell viability, proliferation and migration, as well as epithelial-mesenchymal transition and increased cell apoptosis. Mechanistically, LINC00857 abundantly interacted with the RNA-binding protein YTH domain containing 1 (YTHDC1). YTHDC1 ultimately combined with solute carrier family 7 member 5 (SLC7A5) and increased SLC7A5 mRNA stability. Finally, a series of rescue experiments indicated that LINC00857 promoted the proliferation and migration of CRC cells by regulating mRNA stability. Thus, the present findings illustrated that LINC00857 functions as an oncogene in CRC cells via the YTHDC1/SLC7A5 axis.
\end{abstract}

\section{Introduction}

Colorectal cancer (CRC) is the world's fourth most lethal malignancy, causing $~ 900,000$ mortalities every year (1-4). It is the third most common malignancy in males after prostate and lung cancer, and the second most common malignancy in females after breast cancer (5). Over the last 20 years,

Correspondence to: Dr Ming Xu, Department of Gastrointestinal Surgery, Chenzhou No. 1 People's Hospital, 102 Luojiajing Road, Chenzhou, Hunan 423000, P.R. China

E-mail: xumingno1@hotmail.com

Key words: long intergenic non-protein coding RNA 857, RNA binding protein, YTH domain containing 1, solute carrier family 7 member 5 the incidence of $\mathrm{CRC}$ has been rapidly rising in those aged $<50$ years $(2,4)$. Despite advances in systemic therapy, many patients with metastatic CRC succumb to their disease (6). Therefore, there is an urgent requirement for more effective therapeutic strategies for CRC. It is of great significance to identify biomarkers affecting cell proliferation and migration, and to determine the regulatory mechanisms in CRC cells.

Long non-coding RNAs (lncRNAs) are non-coding transcripts that are $>200$ nucleotides long and have previously been identified as one of the largest and most diverse RNA families $(7,8)$. IncRNAs are modulators involved in a variety of biological processes (9-11). They regulate gene expression via different mechanisms in the cytoplasm or nucleus $(11,12)$. Additionally, RNA-binding proteins (RBPs) exert vital effects on RNA processing and metabolism, including mRNA localization, mRNA stability control and translation control $(13,14)$. The abnormal expression and mutation of RBP genes affect different steps of RNA processing, thereby changing the function of the target gene (13).

RNA-protein interactions create vital checkpoints during the regulation of gene expression at the RNA level (15). lncRNAs are associated with a variety of cellular functions, most of which involve interactions with one or more RBPs (16). RBPs usually bind to numerous different RNAs. Furthermore, RBPs bind to target transcripts and alter their translation or stability (17). IncRNAs interact with specific RBPs to enhance mRNA stability in the cytoplasm $(18,19)$. It has been revealed that lncRNA long intergenic non-protein coding RNA 857 (LINC00857) serves as a tumor promotor in esophageal adenocarcinoma (20), hepatocellular carcinoma (HCC) (21) and bladder cancer (22); however, its role and mechanism in CRC cells remain elusive.

Thus, the aim of the present study was to focus on the role and regulatory mechanism of LINC00857 in CRC cells in order to provide potential novel insight into CRC therapy.

\section{Materials and methods}

Bioinformatics analysis. The interactions between LINC00857, solute carrier family 7 member 5 (SLC7A5) and YTH domain containing 1 (YTHDC1) were predicted using the starBase database (http://starbase.sysu.edu.cn/). Additionally, the extent of binding between LINC00857 and YTHDC1 was predicted 
though the RNA-Protein Interaction Prediction (RPISeq) website (http://pridb.gdcb.iastate.edu/RPISeq/).

Patient sample collection. Forty CRC specimens and adjacent non-tumor tissue samples were obtained from 20 male and 20 female patients (age range, 32-73 years; mean age, $51.4 \pm 6.1$ years) who did not receive therapy before undergoing surgery at Chenzhou No. 1 People's Hospital. The tissue samples were harvested via surgical resection or colonoscopy. Adjacent non-tumor tissue samples were obtained from $\geq 1 \mathrm{~cm}$ away from the tumor tissues. Only histologically confirmed new CRC cases that had not previously been diagnosed for cancer were included in the present study. Tumor location (left- or right-sided CRC) was histologically confirmed. The histological grade was assessed according to World Health Organization criteria (23). Tumors were staged according to the tumor-node-metastasis staging system of the American Joint Committee on Cancer (7th edition) (24). All patients provided written informed consent and the study was approved by the Ethics Committee of the Chenzhou No. 1 People's Hospital (Chenzhou, China). All of the methods included in the present study were performed rigidly according to the approved guidelines. Immediately after surgical resection, the tissue samples were frozen in liquid nitrogen and stored at $-80^{\circ} \mathrm{C}$.

RNA extraction and reverse transcription-quantitative polymerase chain reaction ( $R T-q P C R)$. Total RNA from the tissue samples and cells (CRC cell lines, SW480, SW620, LoVo and LS174T cells and human normal colonic epithelial cell line, FHC) was extracted using TRIzol ${ }^{\circledR}$ reagent (Invitrogen; Thermo Fisher Scientific, Inc.). The RNA quality and concentration were determined using the NanoDrop ${ }^{\mathrm{TM}} 2000$ spectrophotometer (Thermo Fisher Scientific, Inc.) at absorbances of 260 and $280 \mathrm{~nm}$. Total RNA was digested using DNase I (Thermo Fisher Scientific Inc.) for $90 \mathrm{~min}$ at $37^{\circ} \mathrm{C}$ and then reverse transcribed into complementary DNA (cDNA) using a reverse transcription kit (Takara Biotechnology Co., Ltd.) according to the manufacturer's instructions. The cDNA template ( $3 \mu \mathrm{l}$; corresponding to $\sim 150 \mathrm{ng}$ of the extracted total RNA) was prepared prior to each PCR reaction. SYBR ${ }^{\circledR}$ Premix Ex Taq ${ }^{\mathrm{TM}}$ (Takara Biotechnology Co., Ltd.) was used for RT-qPCR analysis, which was performed on an Applied Biosystems $^{\text {TM }} 7500$ Real-Time PCR System. The relative quantification was calculated according to the $2^{-\Delta \Delta \mathrm{Cq}}$ method as previously described (25). The results were normalized to GAPDH expression. Primer sequences used in the present study are presented in Table SI and the following thermocycling conditions were used: Initial denaturation at $95^{\circ} \mathrm{C}$ for $3 \mathrm{~min}$, followed by 40 cycles at $95^{\circ} \mathrm{C}$ for $5 \mathrm{sec}, 60^{\circ} \mathrm{C}$ for $30 \mathrm{sec}$ and at $72^{\circ} \mathrm{C}$ for $45 \mathrm{sec}$, before final extension at $72^{\circ} \mathrm{C}$ for $3 \mathrm{~min}$.

Cell culture and transfection. CRC cell lines, SW480, SW620, LoVo and LS174T cells and a human normal colonic epithelial cell line, FHC were purchased from the American Type Culture Collection. All CRC cells were cultured in Gibco Dulbecco's modified Eagle's medium (DMEM; Thermo Fisher Scientific, Inc.) supplemented with Gibco $10 \%$ fetal bovine serum (FBS; Thermo Fisher Scientific, Inc.), $100 \mathrm{U} / \mathrm{ml}$ penicillin and $100 \mathrm{mg} / \mathrm{ml}$ streptomycin (Invitrogen; Thermo Fisher Scientific, Inc.) in a humidified atmosphere at $37^{\circ} \mathrm{C}$ with $5 \% \mathrm{CO}_{2}$.
Short hairpin (sh)-RNAs for LINC00857 (sh-LINC00857\#1/2) and YTHDC1 (sh-YTHDC1) and a negative control (sh-NC) were transfected into CRC cells. Additionally, the sequence of LINC00857 was synthesized and subcloned into a pcDNA3.1 plasmid (Invitrogen; Thermo Fisher Scientific, Inc.) to overexpress LINC00857 with an empty pcDNA3.1 vector serving as the NC. The sequence of YTHDC1 was synthesized and subcloned into a pcDNA3.1 plasmid to overexpress YTHDC1 with an empty pcDNA3.1 vector serving as the NC. The sequence of SLC7A5 was synthesized and subcloned into a pcDNA3.1 plasmid to overexpress SLC7A5 with an empty pcDNA3.1 vector serving as the NC. SW480 and SW620 cells $\left(1 \times 10^{5}\right.$ cells/well) were seeded in 24 -well plates and $500 \mu 1$ DMEM was added to each well. When the cells reached $40-60 \%$ confluence, the abovementioned vectors were transfected into cells at a final concentration of $50 \mathrm{nM}$ using Lipofectamine ${ }^{\circledR} 2000$ reagent (Invitrogen; Thermo Fisher Scientific, Inc.) at $37^{\circ} \mathrm{C}$ with $5 \% \mathrm{CO}_{2}$ according to the manufacturer's instructions. Cells were harvested using TRIzol (Invitrogen; Thermo Fisher Scientific, Inc.) and transferred to 1.5-ml Eppendorf tubes for mixture and stored at $-80^{\circ} \mathrm{C}$ for further analysis $48 \mathrm{~h}$ post-transfection. Finally, RT-qPCR was performed to evaluate the transfection efficiency. The plasmid sequences used in the present study are presented in Table SI.

Cell counting kit-8 (CCK-8) assay. Following transfection with sh-LINC00857\#1/2 or sh-NC, SW480 and SW620 cells $\left(1 \times 10^{3}\right.$ cells/well) were plated into 96 -well plates. Cell viability was detected on days 0-2 and 3 using a CCK- 8 assay (Dojindo Molecular Technologies, Inc.) according to the manufacturer's instructions. Optical density was assessed at a wavelength of $450 \mathrm{~nm}$ using a microplate reader (Thermo Fisher Scientific, Inc.).

Colony formation assay. For colony formation assays, SW480and SW620-transfected cells $\left(1 \times 10^{3}\right)$ were placed in 6-well plates and cultivated in proper medium supplemented with $10 \%$ FBS for 14 days and, during this period, the medium was replaced every 4 days. After 14 days, the cells were fixed with methanol and stained with $0.1 \%$ crystal violet (MilliporeSigma) for $30 \mathrm{~min}$ at room temperature. Images of the visible colonies were obtained and the number of colonies was counted by a light microscope (OLYMPUS IX-71; Olympus Corporation) (magnification, x10) after $48 \mathrm{~h}$.

5-Ethynyl-2-deoxyuridine (EdU) assay. EdU experiments were conducted with an EdU labeling/detection kit (Guangzhou RiboBio Co., Ltd.) according to the manufacturer's instructions. The transfected cells were placed in $50 \mu \mathrm{M}$ of EdU staining medium and maintained for an additional $2 \mathrm{~h}$ at room temperature. After washing the cells three times with $0.5 \mathrm{~g} / \mathrm{ml}$ phosphate-buffered saline (PBS), the nuclei were stained with DAPI (Invitrogen; Thermo Fisher Scientific, Inc.) for $10 \mathrm{~min}$ in the dark room at room temperature. Finally, fluorescence microscopy was utilized to observe and count EdU-positive cells. The percentage of EdU-positive cells was calculated from five random fields in three wells.

Flow cytometry analysis. Cell apoptosis was assessed via flow cytometry. SW480 and SW620 cells were subjected to double 
staining with fluorescein isothiocyanate-Annexin V and propidium iodide (Becton-Dickinson and Company) according to the manufacturer's instructions. The cells were detected via flow cytometry using a FACScan ${ }^{\circledR}$ (BD Biosciences) equipped with CellQuest Pro software v5.1 (BD Biosciences).

Wound healing assay. Following transfection, SW480 and SW620 $\left(2 \times 10^{5}\right)$ cells seeded in 6-well plates were subjected to serum starvation for $4 \mathrm{~h}$ and cultured to $100 \%$ confluence. Thereafter, a wound was simulated by scratching a straight line in the cell monolayer using a sterile $200-\mu 1$ pipette tip. After gently scraping the scratched monolayer cells twice with serum-free medium (Gibco; Thermo Fisher Scientific Inc.), the wound was healed in complete medium for $24 \mathrm{~h}$. After the wound was formed, the wound widths at 0 and $24 \mathrm{~h}$ were visualized using an inverted microscope. The percentage of wound closure was assessed for cell migration.

Western blot analysis. Total proteins were extracted from cells using radioimmunoprecipitation assay buffer (Beyotime Institute of Biotechnology) including protease inhibitors (Beyotime Institute of Biotechnology) and protein concentrations were evaluated using a bicinchoninic acid Protein Assay Kit (Beyotime Institute of Biotechnology). The protein lysates were separated using 10\% SDS-polyacrylamide gel electrophoresis and equal quantities (5 $\mu \mathrm{g}$ per sample) of separated proteins were transferred to $0.22-\mu \mathrm{m}$ nitrocellulose membranes (MilliporeSigma). After blocking with 5\% non-fat milk for $1 \mathrm{~h}$ at room temperature, the membranes were incubated with specific primary antibodies and treated with specific primary antibodies overnight at $4^{\circ} \mathrm{C}$. GAPDH (cat. no. ab8245; 1:500) served as the internal control. The primary antibodies (Abcam) were as follows: Anti-E-cadherin (cat. no. ab1416; 1:50), anti-N-cadherin (cat. no. ab98952; 1:500), anti-MMP2 (cat. no. ab92536; 1:1,000), anti-MMP9 (cat. no. ab76003; 1:1,000) and anti-SLC7A5 (cat. no. ab99419; 1:1,000). After washing three times with PBS, the membranes were incubated with horseradish peroxidase-conjugated goat anti-rabbit antibodies [cat. no. sc-2357 (1:5,000) Santa Cruz Biotechnology, Inc.] for another $2 \mathrm{~h}$ at room temperature. The proteins were visualized using an enhanced chemiluminescence detection kit (Amersham; Cytiva) and the Odyssey Infrared Imaging system version 2.1 (LI-COR Biosciences).

Subcellular fractionation. Cytoplasmic and nuclear extracts were extracted from SW480 or SW620 cells using NE-PER ${ }^{\text {TM }}$ kit (cat. no. 78833; Thermo Fisher Scientific, Inc.). RT-qPCR analysis was performed to detect the RNAs isolated from the nucleus or cytoplasm. Expression levels of U6, the nuclear control and GAPDH, the cytoplasmic control were evaluated.

RNA immunoprecipitation (RIP) assay. The RIP assay was conducted using a Magna RIP ${ }^{\mathrm{TM}}$ RNA-Binding Protein Immunoprecipitation Kit (MilliporeSigma) according to the manufacturer's instructions. CRC cells at $80-90 \%$ confluency were scraped off and lysed in complete RIP lysis buffer. Whole cell extracts $(100 \mu \mathrm{l})$ were cultivated with magnetic beads for $6 \mathrm{~h}$ at $4^{\circ} \mathrm{C}$ and the beads were conjugated with anti-UPF1 RNA helicase and ATPase (UPF1), anti-heterogeneous nuclear ribonucleoprotein C (HNRNPC), anti-YTHDC1 or control
IgG. After the magnetic beads were washed three times, Proteinase K (Thermo Fisher Scientific Inc.) was applied to remove proteins from the complexes. Purified RNAs were subjected to RT-qPCR analysis.

In vitro transcription and RNA pull-down assay. T7 RNA polymerase (Thermo Fisher Scientific Inc.) was utilized to transcribe LINC00857 in vitro (YTHDC1), a RNeasy Plus Mini Kit (Qiagen Sciences, Inc.) was utilized to purify LINC00857 (YTHDC1) and RNase-free DNase I (Qiagen Sciences, Inc.) was utilized to treat LINC00857 (YTHDC1). The transcribed LINC00857 (YTHDC1) was labeled with Biotin RNA Labeling Mix (Thermo Fisher Scientific Inc.) and the RNA pull-down assays were performed with a Pierce ${ }^{\mathrm{TM}}$ Magnetic RNA-Protein Pull-Down Kit (Pierce; Thermo Fisher Scientific, Inc.) according to the manufacturer's instructions.

RNA stability assay. Following transfection, CRC cells were treated with $1 \mu \mathrm{g} / \mathrm{ml}$ actinomycin D. Next, the cells were collected at different time points (0,3,6 and $9 \mathrm{~h})$ and RNA was extracted with TRIzol ${ }^{\mathrm{TM}}$ reagent (Invitrogen; Thermo Fisher Scientific, Inc.). Finally, RT-qPCR analysis was performed to evaluate the mRNA levels.

Statistical analysis. Each experiment was repeated three times. Both paired and unpaired Student's t-test were used to analyze differences between two groups. Paired Student's t-test was used for RT-qPCR analysis to evaluate gene expression in CRC tissues and paired adjacent non-tumor tissue samples. One-way analysis of variance followed by Tukey's post hoc test was used to analyze the differences between three groups. All statistical analyses were performed with SPSS v.20.0 software (IBM Corp.) and the data are presented as the means \pm standard deviation. $\mathrm{P}<0.05$ was considered to indicate a statistically significant difference.

\section{Results}

Significantly upregulated LINC00857 in CRC cells promotes CRC cell proliferative and migratory abilities in vitro. According to previous studies, LINC00857 expression is upregulated in certain types of cancer (20-22). Therefore, to verify the abnormal expression of LINC00857 during CRC progression, RT-qPCR analysis was performed with CRC and adjacent healthy tissue samples, as well as with four CRC cell lines (SW480, SW620, LoVo and LS174T) and a human normal colonic epithelial cell line (FHC). The results indicated that LINC00857 was significantly upregulated in CRC tissue samples (Fig. 1A). Consistently, LINC00857 expression was higher in CRC cells than in healthy cells (Fig. 1B). Additionally, the RT-qPCR results demonstrated a close association between LINC00857 and the right-sided CRC samples (Fig. S1A). There was a statistically significant association between high LINC00857 expression and the right-sided CRC samples (Table I). Furthermore, LINC00857 expression was observed to be upregulated in advanced stage CRC tissue samples (Fig. S1B). In addition, higher LINC00857 expression was closely associated with larger tumor size, more advanced stage and poor histological grade (Table I). 

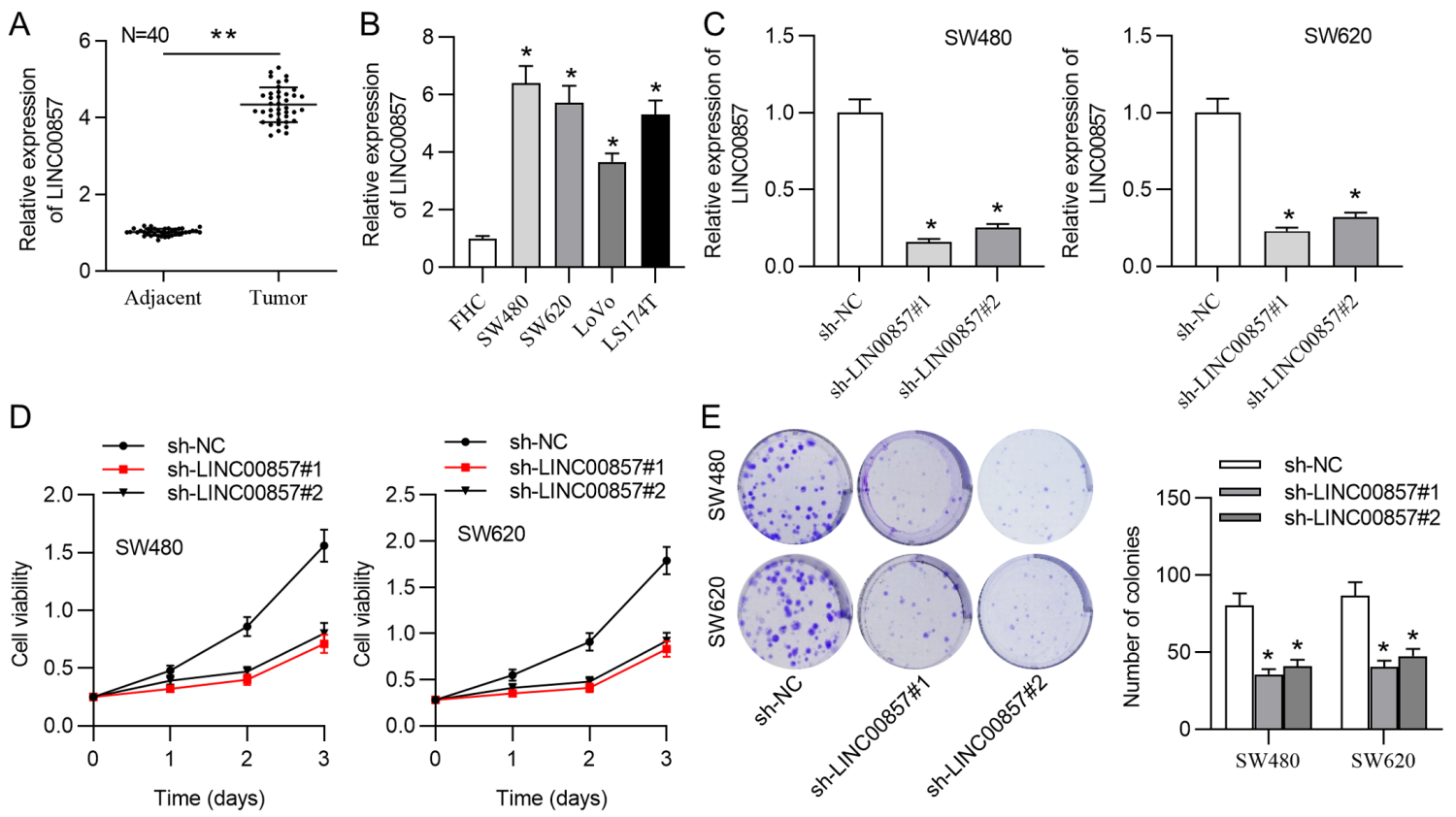

F
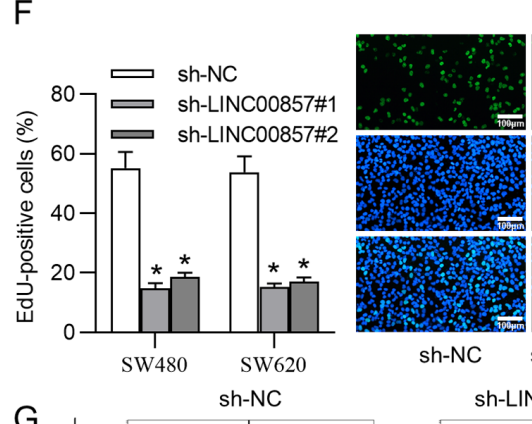

SW480
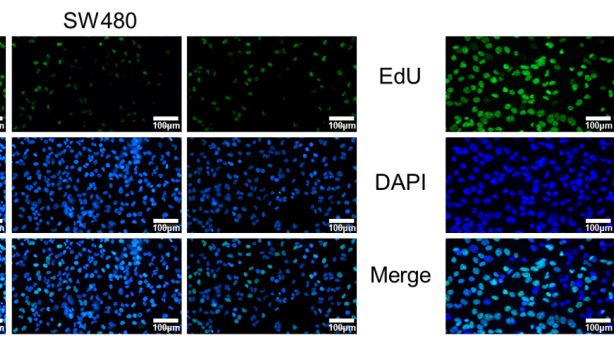

Sw620

G

sh-NC sh-LINC0085
sh-LINC00857\#1

sh-NC
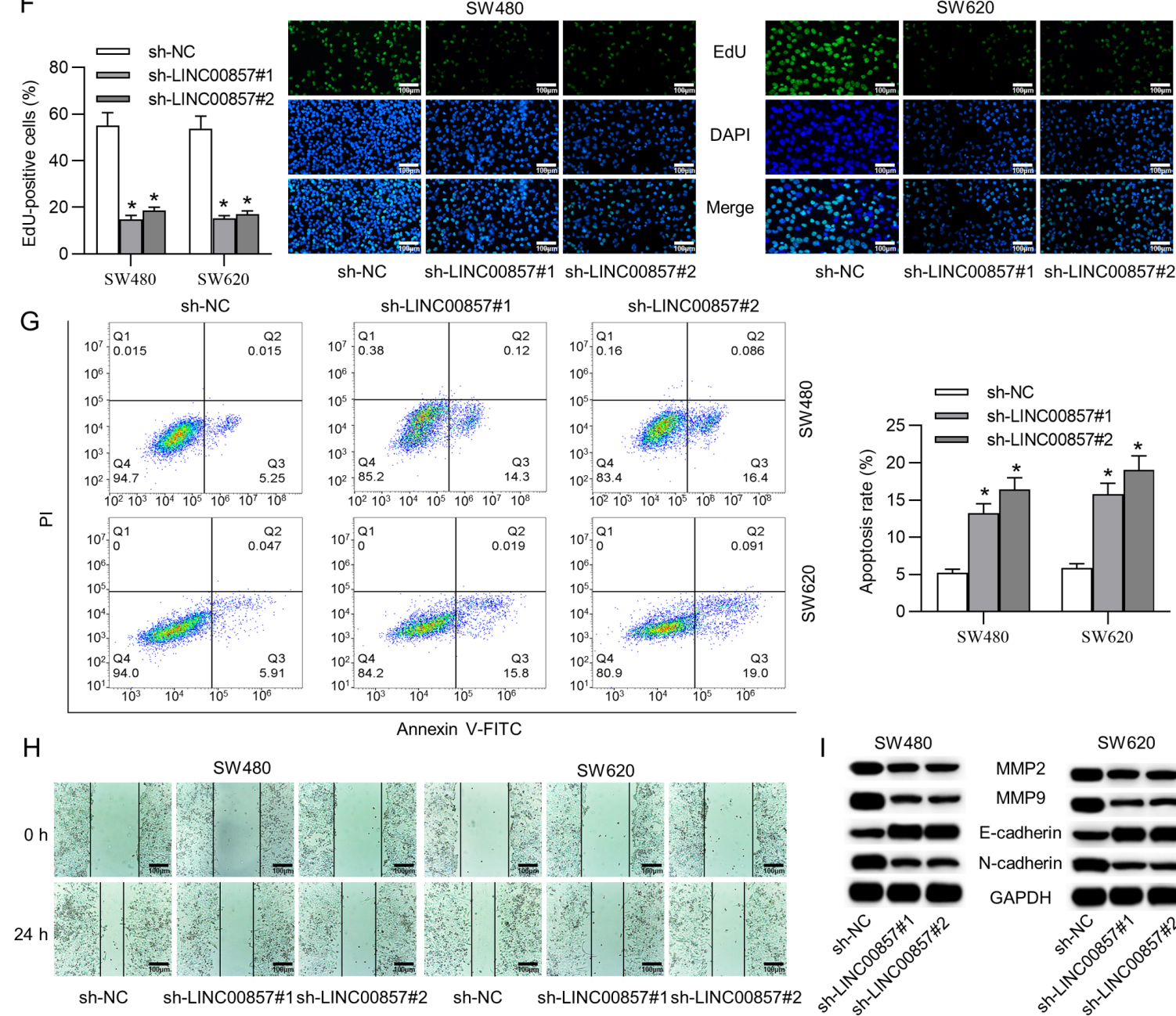

Figure 1. Expression level of LINC00857 in CRC tissue samples and cells. (A) RT-qPCR analysis of LINC00857 levels in human CRC and adjacent tissue samples. ${ }^{* * *} \mathrm{P}<0.01$. (B) RT-qPCR analysis of LINC00857 levels in the FHC and CRC cell lines. "P<0.05 vs. FHC. (C) LINC00857 levels in SW480 and SW620 cells transfected with sh-NC or sh-LINC00857\#1/2 were examined by RT-qPCR. "P $<0.05$ vs. sh-NC. (D) Cell Counting Kit- 8 assay determined the viability of sh-LINC00857-transfected CRC cells. (E) Colony formation assay confirmed the proliferative ability of SW480 and SW620 cells under LINC00857 downregulation. "P<0.05 vs. sh-NC. (F) Proliferating SW480 and SW620 cells were EdU-stained and cell nuclei were stained with DAPI (scale bar, $100 \mu \mathrm{m}$ ). ${ }^{*} \mathrm{P}<0.05$ vs. sh-NC. (G) The apoptotic rates of SW480 and SW620 cells after LINC00857 knockdown were confirmed by flow cytometric analysis. "P $<0.05$ vs. sh-NC. (H) Wound healing assays verified the changes in the migratory ability of CRC cells affected by silencing LINC00857 (scale bar, $100 \mu \mathrm{m}$ ). (I) Migration and epithelial-mesenchymal transition were further confirmed by western blot under LINC00857 knockdown. LINC00857, long intergenic non-protein coding RNA 857; CRC, colorectal cancer; RT-qPCR, reverse transcription-quantitative PCR; sh, short hairpin; NC, negative control; EdU, 5-ethynyl-2-deoxyuridine. 
Table I. Association between LINC00857 expression and clinical features of 40 colorectal cancer tissue samples.

\begin{tabular}{|c|c|c|c|c|}
\hline \multirow[b]{2}{*}{ Clinicopathological characteristic } & \multirow[b]{2}{*}{ Patients, $\mathrm{n}$} & \multicolumn{2}{|c|}{ LINC00857 expression } & \multirow[b]{2}{*}{ P-value } \\
\hline & & High, n (\%) & Low, n (\%) & \\
\hline Localization & & & & $\mathrm{P}<0.001$ \\
\hline Left & 11 & $2(18.2)$ & $9(81.8)$ & \\
\hline Right & 29 & $24(82.8)$ & $5(17.2)$ & \\
\hline Tumor size (cm) & & & & $\mathrm{P}<0.01$ \\
\hline$\leq 2$ & 14 & $4(28.6)$ & $10(71.4)$ & \\
\hline$>2$ & 26 & $21(80.8)$ & $5(19.2)$ & \\
\hline Clinical T stage & & & & $\mathrm{P}<0.001$ \\
\hline $\mathrm{T} 1-\mathrm{T} 2$ & 13 & $2(15.4)$ & $11(84.6)$ & \\
\hline $\mathrm{T} 3-\mathrm{T} 4$ & 27 & $20(74.1)$ & $7(25.9)$ & \\
\hline Histological grade & & & & $\mathrm{P}<0.01$ \\
\hline Well differentiated & 5 & $1(20.0)$ & $4(80.0)$ & \\
\hline Moderately differentiated & 15 & $4(26.7)$ & $11(73.3)$ & \\
\hline Poorly differentiated & 20 & $17(85.0)$ & $3(15.0)$ & \\
\hline
\end{tabular}

LINC00857, long intergenic non-protein coding RNA 857.

To investigate the role of LINC00857 in CRC cells, SW480 and SW620 cells were transfected with sh-LINC00857\#1/2 or sh-NC vectors to silence LINC00857 and a series of loss-of-function experiments were subsequently performed. The transfection efficiency was analyzed by RT-qPCR and the results indicated that LINC00857 expression was significantly decreased in the SW480 and SW620 cells (Fig. 1C). Furthermore, the CCK-8 assay revealed that cell viability was impaired by LINC00857 downregulation (Fig. 1D). Moreover, the colony formation assay demonstrated a large decrease in the number of colonies (Fig. 1E). Additionally, the EdU staining results illustrated that the percentage of EdU-positive cells in the sh-LINC00857\#1/2-mediated group was diminished (Fig. 1F). These findings cumulatively indicate that silencing LINC00857 suppressed the proliferative ability of CRC cells.

Subsequently, to investigate whether apoptosis regulation was propelled by LINC00857 silencing, flow cytometric analysis was conducted. The results demonstrated that LINC00857 knockdown induced CRC cell apoptosis (Fig. 1G). Additionally, to estimate the effect of LINC00857 knockdown on CRC cell migratory ability, a wound healing assay was performed. The number of migrated CRC cells was decreased following LINC00857 depletion (Fig. 1H). To validate the function of LINC00857 silencing on CRC cell migration, western blot analyses were conducted to evaluate the MMP2, MMP9, E-cadherin and N-cadherin levels. The results showed that the protein levels of MMP2 and MMP9 were markedly decreased after LINC00857 silencing. Simultaneously, the E-cadherin protein level was upregulated and the $\mathrm{N}$-cadherin protein level was downregulated in the sh-LINC00857\#1/2 groups (Fig. 1I).

LINC00857 interacts with RNA-binding protein YTHDC1. The localization of lncRNAs within the cell primarily determines their molecular functions (26). Therefore, to clarify the potential mechanism of LINC00857 in CRC cells, subcellular localization was conducted. The results indicated LINC00857 was primarily distributed in the cytoplasm of CRC cells (Fig. 2A) indicating that LINC00857 post-transcriptionally regulated gene expression. Cytoplasmic lncRNAs cooperate with RBPs in different types of cancer (27). Therefore, it was hypothesized that LINC00857 interacts with RBPs to regulate CRC cells. Six potential RBPs were predicted using the starBase database (Fig. 2B). The interaction between LINC00857 and these candidate RBPs was evaluated by RPISeq. The Random Forest classifier and Support Vector Machine classifier scores were $>0.5$, indicating that LINC00857 may bind to UPF1, YTHDC1 and HNRNPC (Fig. 2C). To determine the interaction between these RBPs and LINC00857 an RIP assay was performed. LINC00857 presented a significant enrichment conjugated with anti-YTHDC1 rather than anti-UPF1 or anti-HNRNPC (Fig. 2D). Similarly, high binding affinity between LINC00857 and YTHDC1 was verified via western blotting performed following the RNA pull-down assay. The results demonstrated significant enrichment in pull-down products by biotinylated LINC00857 sense and no enrichment was observed in pull-down products by biotinylated LINC00857 antisense by blotting using the anti-YTHDC1 antibody (Fig. 2E). Furthermore, according to RT-qPCR results, YTHDC1 was upregulated in CRC tissue samples and cells (Fig. 2F and G).

LINC00857 was transfected with the pcDNA3.1/ LINC00857 plasmid and RT-qPCR revealed that LINC00857 expression was significantly increased in the CRC cells (Fig. 2H). RT-qPCR was also used to examine the transfection efficiency of pcDNA3.1/YTHDC1 and sh-YTHDC1. The results revealed that YTHDC1 was upregulated in the pcDNA3.1/YTHDC1 group and downregulated in the sh-YTHDC1 group (Fig. 2I). YTHDC1 expression was not altered upon LINC00857 silencing or elevation and LINC00857 expression was not changed upon 
A

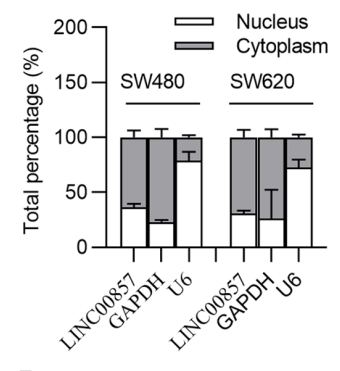

D

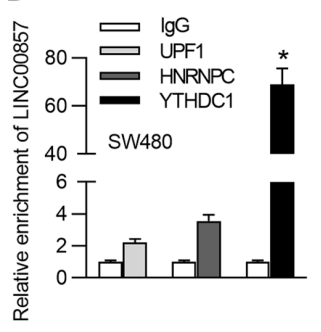

$\mathrm{G}$
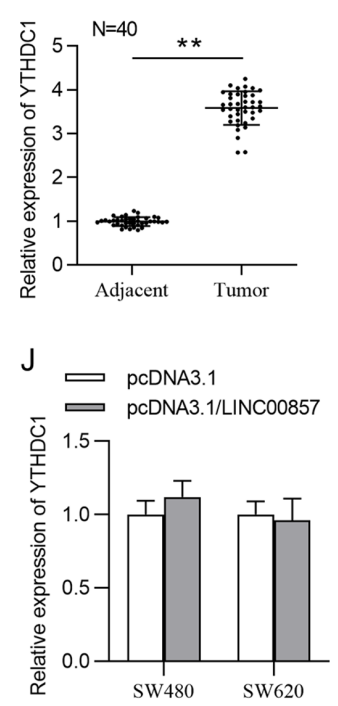

B

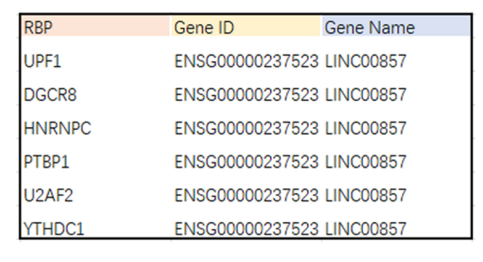

C

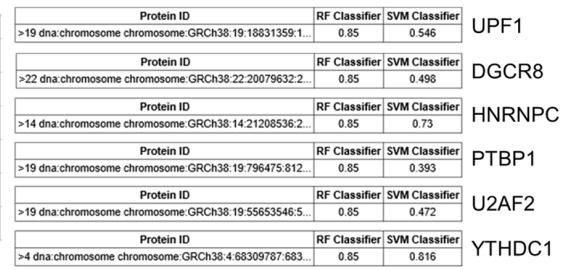

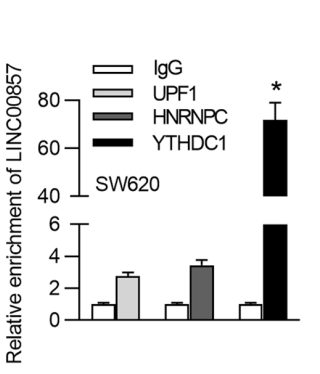

$\mathrm{H}$
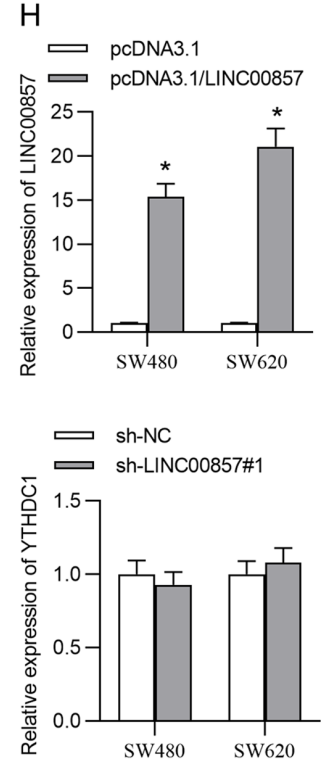

E

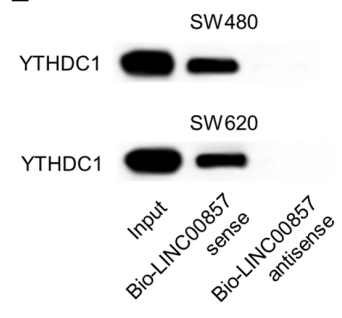

I
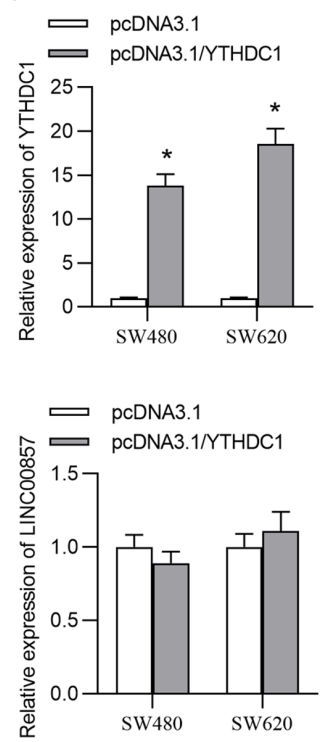
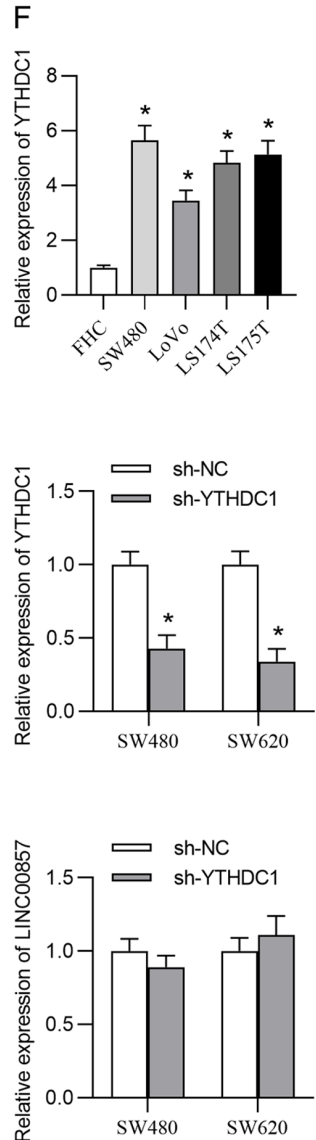

Figure 2. Interaction between LINC00857 and RBP YTHDC1. (A) LINC00857 localization was determined through subcellular fractionation. U6 served as a nuclear marker and GAPDH as a cytosolic marker. (B) RBPs that LINC00857 potentially bound to were obtained using the starBase database. (C) The data indicating a possible interaction between LINC00857 and YTHDC1 were acquired from the RNA-protein interaction prediction website. Scores $>0.5$ were regarded as positive, implying the high possibility of RNA and protein binding. (D) RNA immunoprecipitation experiments were conducted in SW480 and SW620 cells to evaluate the enrichment of LINC00857 in each group. "P<0.05 vs. IgG. (E) Biotinylated LINC00857 pulled down YTHDC1, as demonstrated by western blot analysis. (F) RT-qPCR was used to determine YTHDC1 expression in CRC and control cells. "P<0.05 vs. FHC. (G) LINC00857 levels were examined in CRC and adjacent tissue samples using RT-qPCR. ${ }^{* *} \mathrm{P}<0.01$. $(\mathrm{H})$ LINC00857 mRNA levels were determined through RT-qPCR under upregulated LINC00857 in CRC cells. " $\mathrm{P}<0.05$ vs. pcDNA3.1 (I) RT-qPCR confirmed the YTHDC1 expression level to determine the transfection efficacy of pcDNA3.1/YTHDC1 or sh-YTHDC1. "P<0.05 vs. pcDNA3.1. (J) The expression level of LINC00857 in CRC cells was evaluated under YTHDC1 silencing and overexpression. LINC00857, long intergenic non-protein coding RNA 857; RBP, RNA-binding proteins; YTHDC1, YTH domain containing 1; RT-qPCR, reverse transcription-quantitative PCR; CRC, colorectal cancer; sh, short hairpin; NC, negative control.

YTHDC1 upregulation or downregulation (Fig. 2J). These findings indicated that YTHDC1 and LINC00857 cannot mutually affect gene expression, verifying that they were bound together.

LINC00857 increases SLC7A5 stability by recruiting YTHDC1. Previous studies reported that RBPs interact with mRNAs and increase mRNA stability (28-30). The present study hypothesized whether YTHDC1 had a similar regulatory function on its target in CRC cells. First, 10 potential target mRNAs were predicted using the starBase database
(Fig. 3A). Among all the putative mRNAs, SLC7A5, plectin (PLEC) and zinc finger homeobox 3 (ZFHX3) were successfully confirmed with western blotting of complexes pulled down by biotinylated YTHDC1 (Fig. 3B). RT-qPCR results demonstrated that SLC7A5 expression decreased under YTHDC1 downregulation, and PLECs and ZFHX3 were not decreased (Fig. 3C). SLC7A5 was therefore identified as a downstream target of YTHDC1 and selected for subsequent experiments. Subsequently, SLC7A5 expression in CRC tissue samples and cells was evaluated using RT-qPCR. 
A

\begin{tabular}{|c|c|}
\hline RBP & Gene Name \\
\hline YTHDC1 & ENSG00000103275 SLC7A5 \\
\hline YTHDC1 & ENSG00000178209 PLEC \\
\hline YTHDC1 & ENSG00000155506 LARP1 \\
\hline YTHDC1 & ENSG00000140836 ZFHX3 \\
\hline YTHDC1 & ENSG00000167522 ANKRD11 \\
\hline YTHDC1 & ENSG00000167978 SRRM2 \\
\hline YTHDC1 & ENSG00000140443 IGF1R \\
\hline YTHDC1 & ENSG00000204469 PRRC2A \\
\hline YTHDC1 & ENSG00000086758 HUWE1 \\
\hline YTHDC1 & ENSG00000130723 PRRC2B \\
\hline
\end{tabular}

C

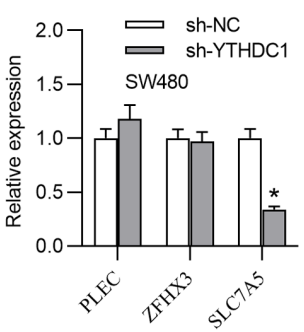

$\mathrm{F}$
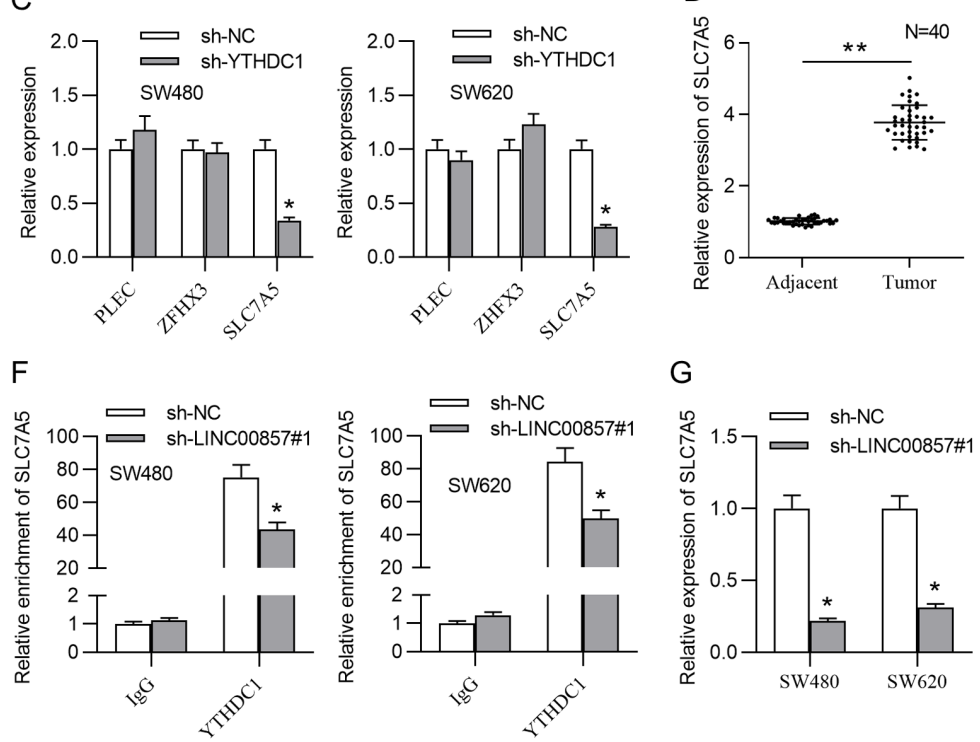

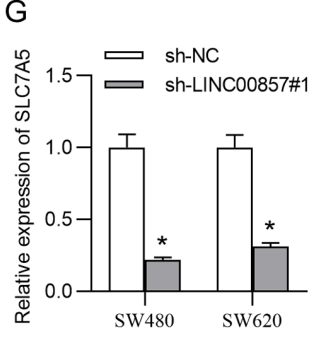

B
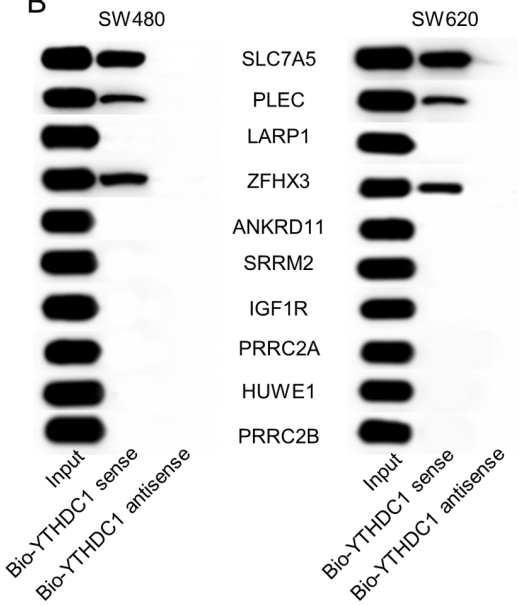

D

$\mathrm{E}$
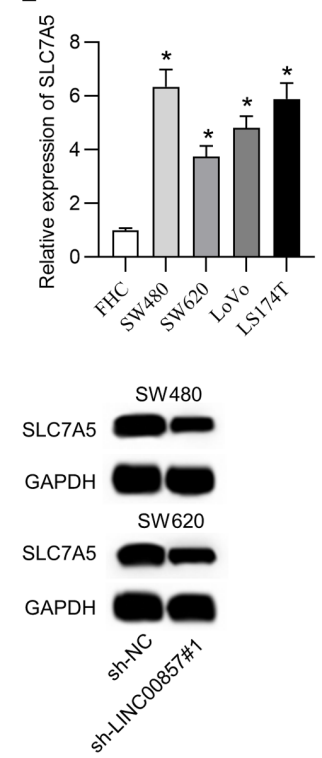

$\mathrm{H}$
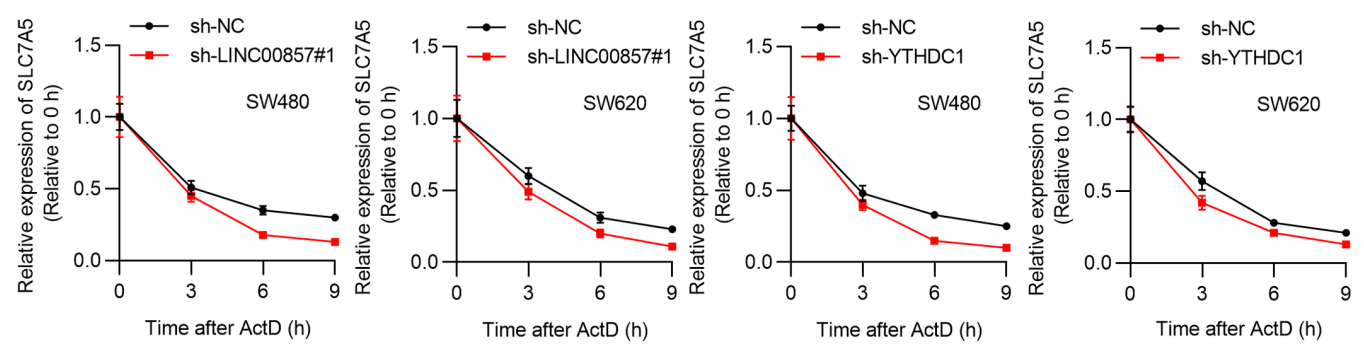

Figure 3. SLC7A5 was a potential downstream target of LINC00857-YTHDC1. (A) Putative targets of YTHDC1 were acquired from the starBase database. (B) Western blot analysis verified the identity of the mRNAs pulled down by biotinylated YTHDC1. (C) The expression of the candidate mRNAs after YTHDC1 knockdown was evaluated via RT-qPCR analysis. (D) RT-qPCR detected SLC7A5 levels in CRC and adjacent tissue samples. ${ }^{* *} \mathrm{P}<0.01$. (E) YTHDC1 expression was analyzed via RT-qPCR in CRC cell lines and a control cell line. ${ }^{*} \mathrm{P}<0.05$ vs. FHC. (F) RIP experiments verified SLC7A5 enrichment in YTHDC1 RIP under LINC00857 silencing in CRC cells. "P<0.05 vs. sh-NC. (G) SLC7A5 mRNA and protein levels were confirmed after LINC00857 knockdown in CRC cells through RT-qPCR and western blot analysis. " $\mathrm{P}<0.05$ vs. sh-NC. (H) RNA stability assay assessed the degradation rate of SLC7A5 mRNA in CRC cells upon LINC00857 or YTHDC1 knockdown. SLC7A5, solute carrier family 7 member 5; LINC00857, long intergenic non-protein coding RNA 857; YTHDC1, YTH domain containing 1; RT-qPCR, reverse transcription-quantitative PCR; CRC, colorectal cancer; RIP, RNA immunoprecipitation; sh, short hairpin; NC, negative control; PLEC, plectin; ZFHX3, zinc finger homeobox 3; ActD, actinomycin D.

SLC7A5 was found to be significantly upregulated in CRC tissue samples and cells (Fig. 3D and E). Furthermore, the RIP assay with SW480 and SW620 cells showed that the interaction of YTHDC1-SLC7A5 was suppressed under LINC00857 deficiency (Fig. 3F). Furthermore, mRNA and protein expression levels of SLC7A5 decreased as a result of LINC00857 knockdown (Fig. 3G).
These findings further supported LINC00857-YTHDC1 binding and their coregulation of SLC7A5 expression. Additionally, to further probe whether LINC00857-YTHDC1 regulates SLC7A5 mRNA stability,SW480 and SW620 cells were treated with actinomycin D and the decay of pre-existing mRNA was evaluated. The results revealed that silencing LINC00857 or YTHDC1 repressed the SLC7A5 mRNA half-life (Fig. 3H). 

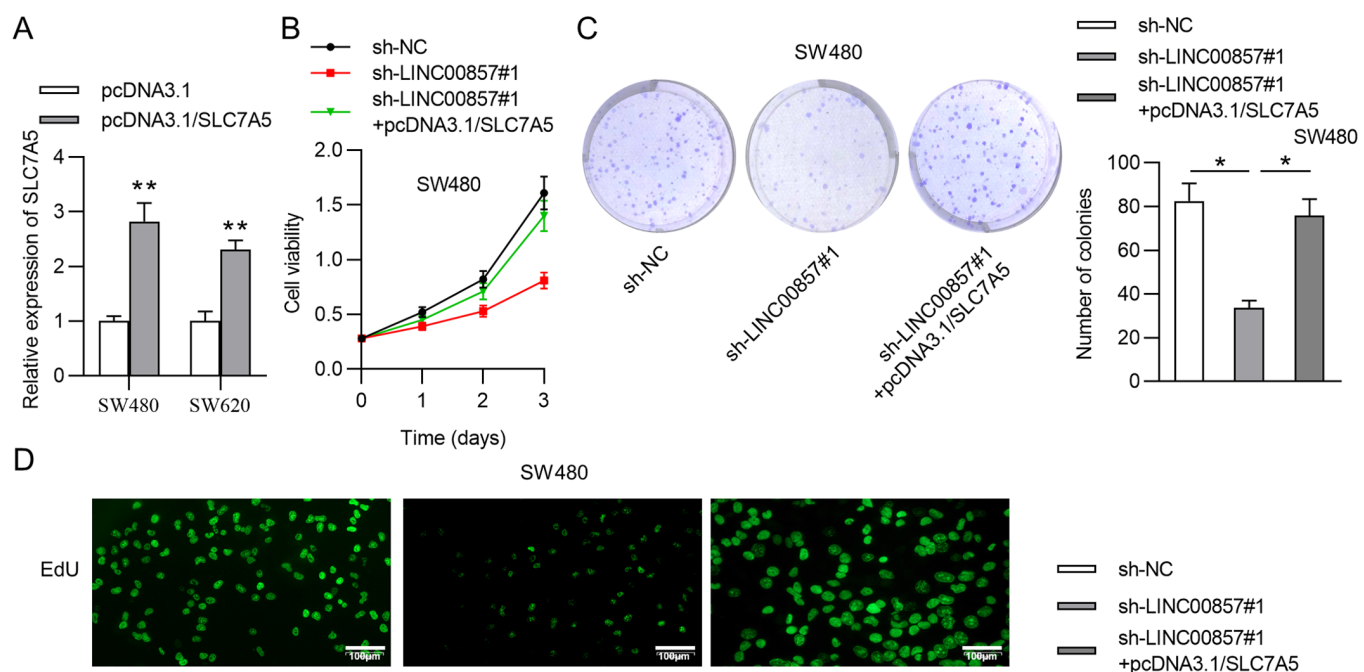

SW 480
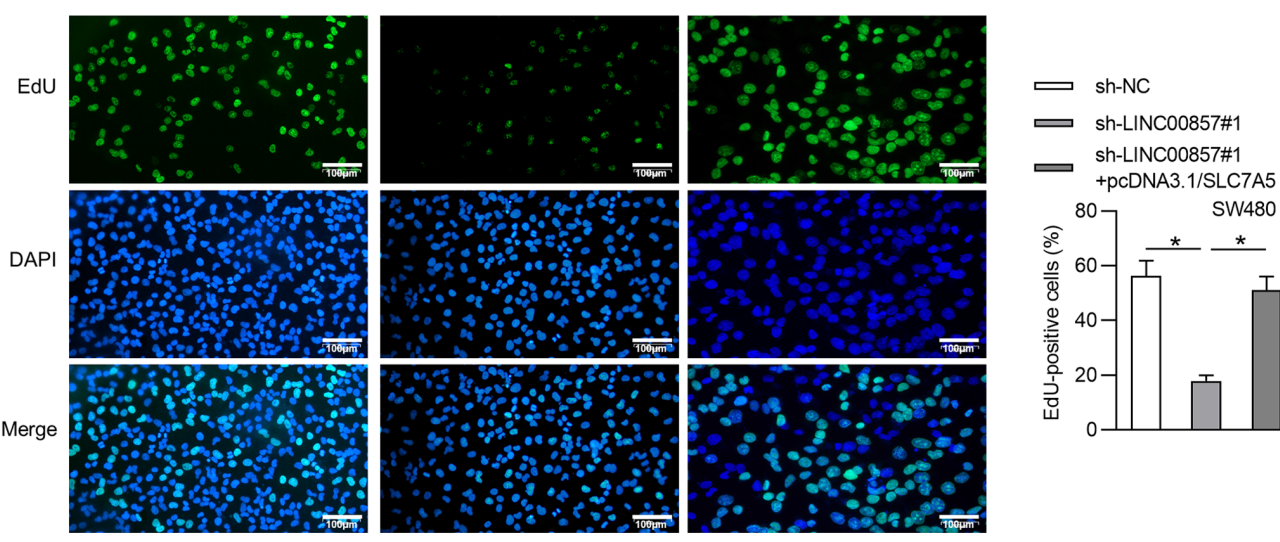

sh-NC

sh-LINC00857\#1
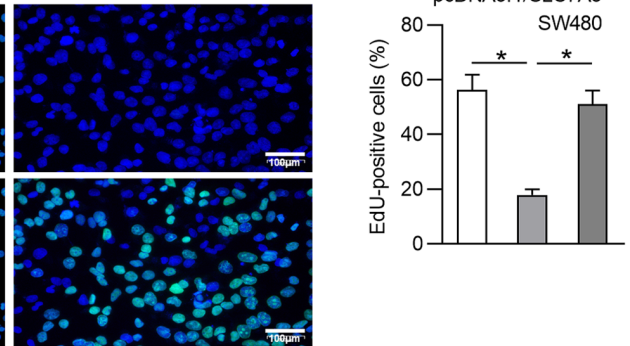

$\mathrm{E}$
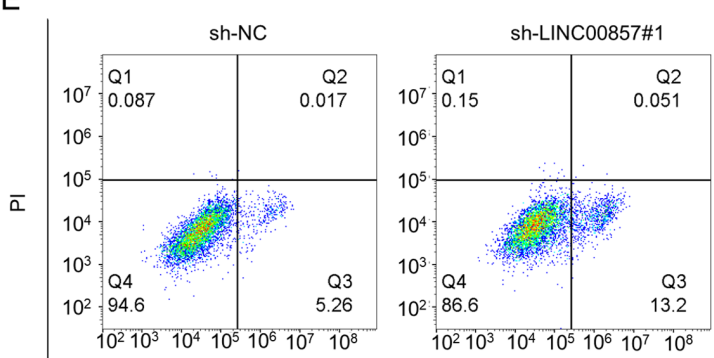

Sh-LINC00857\#1
+ pCDNA3.1/SLC7A5
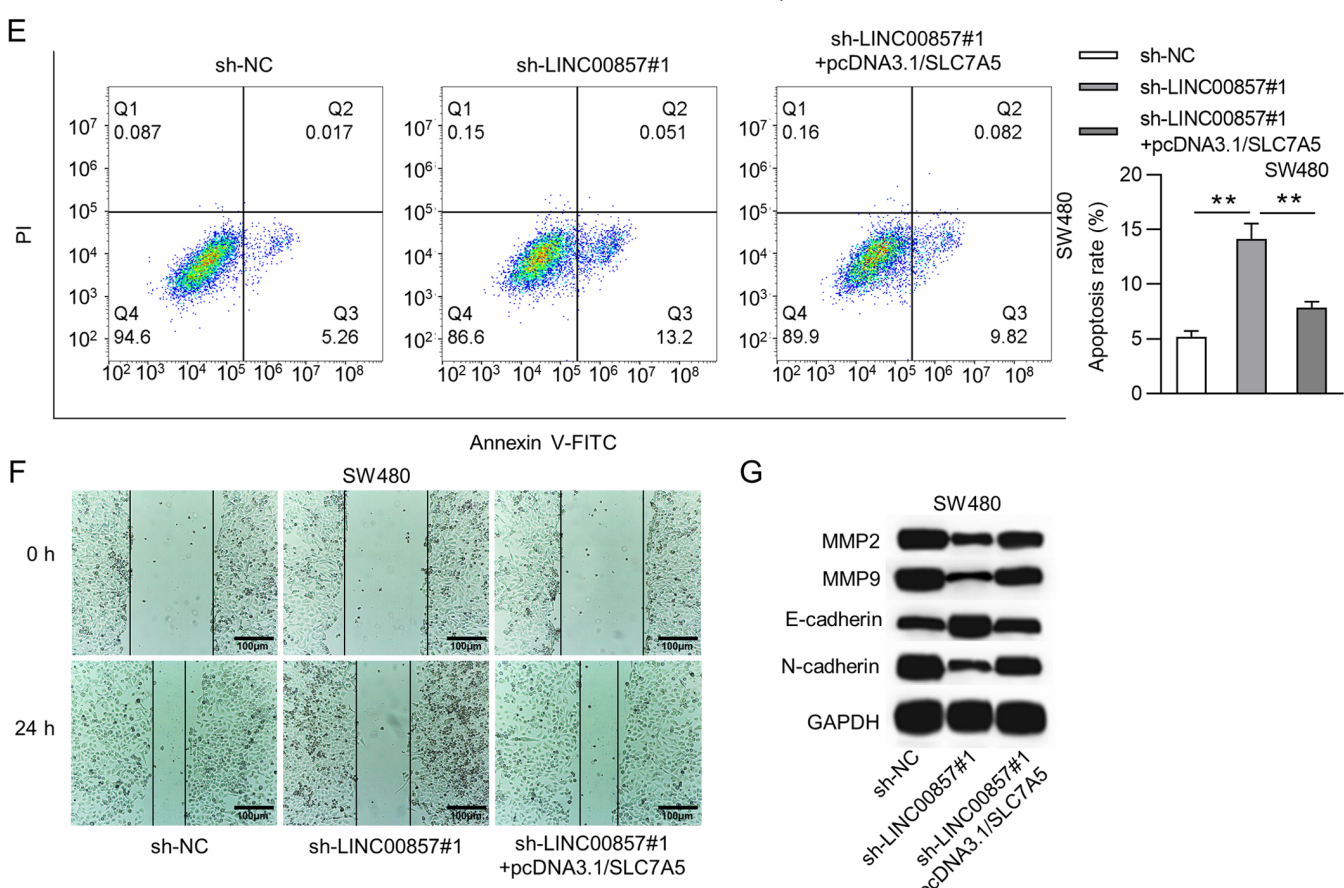

G

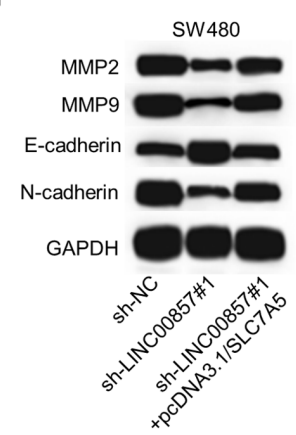

Figure 4. LINC00857 regulated the proliferation and migration of CRC cells by targeting SLC7A5. (A) RT-qPCR analysis demonstrated the transfection efficacy of pcDNA3.1/SLC7A5 in CRC cells. " P<0.01 vs. pcDNA3.1. (B) CRC cells were transfected as indicated and viability was evaluated via CCK-8. (C) A colony formation assay validated the proliferative ability of $\mathrm{CRC}$ cells in each group. " $\mathrm{P}<0.05$. (D) EdU assay confirmed the proliferative ability of $\mathrm{CRC}$ cells in each group (scale bar, $100 \mu \mathrm{m}$ ). $\mathrm{P}<0.05$. (E) Flow cytometric analysis confirmed the cell apoptosis rate in each group. ${ }^{* *} \mathrm{P}<0.01$. (F) The migratory capacity of CRC cells upon the indicated transfection was evaluated by wound healing assay (scale bar, $100 \mu \mathrm{m}$ ). (G) Western blot analysis assessed the protein levels of apoptosis markers under each transfection condition. LINC00857, long intergenic non-protein coding RNA 857; CRC, colorectal cancer; RT-qPCR, reverse transcription-quantitative PCR; EdU, 5-ethynyl-2-deoxyuridine; sh, short hairpin; NC, negative control.

LINC00857 enhances CRC cell proliferative and migratory abilities by upregulating SLC7A5 stability. To probe SLC7A5 involvement in LINC00857-mediated CRC cell proliferation and migration, rescue assays were performed.
CRC cells were co-transfected with sh-LINC00857\#1 and pcDNA3.1/SLC7A5. RT-qPCR analysis indicated that SLC7A5 expression was significantly increased in CRC cells transfected with pcDNA3.1/SLC7A5 (Fig. 4A). The CCK-8 
assay indicated that upregulated SLC7A5 had the opposite effect on CRC cell viability suppressed by LINC00857 silencing (Fig. 4B). Additionally, the proliferation assay results revealed that SLC7A5 overexpression attenuated the decrease in CRC cell proliferative ability under LINC00857 knockdown (Fig. 4C and D). The proportion of apoptotic CRC cells was significantly decreased after co-transfection (Fig. 4E). Furthermore, according to the wound healing assay, CRC cell migration was repressed by LINC00857 depletion, but was recovered under SLC7A5 upregulation (Fig. 4F). Similarly, the western blot analysis revealed that pcDNA3.1/SLC7A5 counteracted the inhibitory effect of LINC00857 silencing on the levels of MMP2, MMP9, E-cadherin and N-cadherin in CRC cells (Fig. 4G).

\section{Discussion}

It has been previously reported that LINC00857 has an oncogenic effect on certain types of cancer, such as esophageal adenocarcinoma (20) and HCC (21). In the present study, LINC00857 was significantly upregulated in CRC tissue samples and cells. Additionally, a series of loss-of-function experiments further validated that LINC00857 knockdown repressed $\mathrm{CRC}$ cell proliferative and migratory abilities and the epithelial-mesenchymal transition (EMT), as well as promoted cell apoptosis in vitro. These data suggested that LINC00857 acted as an oncogene by promoting cell proliferation, migration and EMT during CRC progression.

YTHD-containing proteins participate in numerous RNA processes, such as mRNA splicing, nuclear export, translation and decay in post-transcriptional regulation (31). In eukaryotes, YTHD-containing proteins comprise five functional genes, including YTHDC1 (31). One of the marked effects of YTHDC1 is RNA nuclear export (32). It has previously been demonstrated that the upregulation of YTHDC1 acts as a diagnostic marker, therapeutic target or oncogene in colon cancer (33), HCC (34) and pancreatic adenocarcinoma (35). Additionally, its downregulation indicates a favorable prognosis in patients with head and neck squamous cell carcinoma (36). Notably, YTHDC1 is abundantly expressed in colon adenocarcinoma tissues (37). The localization of lncRNAs within the cell is the primary determinant of their molecular functions (26).

In the present study, the cytoplasmic localization of LINC00857 in CRC cells indicated the post-transcriptional effect of LINC00857 on gene expression. Furthermore, RBPs exert crucial effects by post-transcriptionally regulating gene expression (28), including pre-mRNA splicing, mRNA stabilization, mRNA localization and translation $(17,38)$. The present study hypothesized the potential regulatory pattern of LINC00857 in which LINC00857 interacted with RBPs to stabilize mRNA. The bioinformatics analysis demonstrated that YTHDC1 potentially bound to LINC00857. Subsequent mechanistic experiments verified the binding ability between lncRNA LINC00857 and RBP YTHDC1 in CRC cells. In addition, YTHDC1 expression in CRC tissue samples and cells was identified to be significantly higher than that in normal CRC tissue samples and cells. LINC00857 and YTHDC1 did not mutually affect expression in CRC cells. These findings indicated that LINC00857 interacted with YTHDC1 in CRC cells.
Systematically, SLC7A5 mRNA was demonstrated to interact with YTHDC1 in CRC cells. SLC7A5 (also termed LAT1), characterized as an amino acid transporter, has been revealed to be associated with various types of cancer. For example, SLC7A5 serves as a novel biomarker for high-grade malignancy in prostate cancer (39). Another study demonstrated that positive expression of SLC7A5 predicts poor prognosis in patients with non-small cell lung cancer (40). Furthermore, it has been reported that RBPs post-transcriptionally interact with target mRNAs, thereby strengthening mRNA stability (28-30). In the present study, SLC7A5 was upregulated in CRC tissue samples and cells. Mechanistically, LINC00857 and YTHDC1 increased SLC7A5 mRNA stability in CRC cells. LINC00857 was observed to cooperate with YTHDC1 to stabilize SLC7A5 in $\mathrm{CRC}$ cells. Additionally, rescue assays further verified that the decreased cell proliferation and migration under LINC00857 knockdown was rescued by upregulation of SLC7A5.

However, the present study had several limitations, such as that the results were not validated in vivo. Animal experiments should be performed to further verify in vitro results. In addition, LINC00857 has been reported to competitively bind with miRNAs to regulate mRNA (41). Therefore, other mechanisms mediated by LINC00857 in CRC cells should be explored in the future.

Thus, the present study confirmed that LINC00857, YTHDC1 and SLC7A5 were upregulated in CRC tissue samples and cells. Furthermore, LINC00857 increased CRC cell proliferative and migratory abilities in vitro. The present study hypothesized that LINC00857 abundantly bound to YTHDC1 in CRC cells and that the LINC00857-YTHDC1 complex increased SLC7A5 stability in CRC cells. The present findings may provide novel insight into lncRNA regulatory characteristics in CRC and a novel direction for CRC treatment.

\section{Acknowledgements}

Not applicable.

\section{Funding}

No funding was received.

\section{Availability of data and materials}

The datasets used and/or analyzed during the current study are available from the corresponding author on reasonable request.

\section{Authors' contributions}

ST conceived and designed the experiments. ST, QL, and MX performed the experiments. ST and MX analyzed the data. ST and MX drafted the manuscript. ST and MX confirm the authenticity of all the raw data. All authors read and approved the final version of the manuscript.

\section{Ethics approval and consent to participate}

All patients provided written informed consent and the study was approved by the Ethics Committee of the Chenzhou No. 1 People's Hospital (approval no. 2019-039; Chenzhou, China). 


\section{Patient consent for publication}

Not applicable.

\section{Competing interests}

The authors declare that they have no competing interests.

\section{References}

1. Dekker E, Tanis PJ, Vleugels JLA, Kasi PM and Wallace MB: Colorectal cancer. Lancet 394: 1467-1480, 2019.

2. Patel SG and Ahnen DJ: Colorectal cancer in the young. Curr Gastroenterol Rep 20: 15, 2018.

3. Weitz J, Koch M, Debus J, Höhler T, Galle PR and Büchler MW: Colorectal cancer. Lancet 365: 153-165, 2005.

4. Connell LC, Mota JM, Braghiroli MI and Hoff PM: The rising incidence of younger patients with colorectal cancer: Questions about screening, biology, and treatment. Curr Treat Options Oncol 18: 23, 2017.

5. Castells A: Hereditary forms of colorectal cancer. Gastroenterol Hepatol 39 (Suppl 1): S62-S67, 2016 (In English, Spanish).

6. Wrobel P and Ahmed S: Current status of immunotherapy in metastatic colorectal cancer. Int J Colorectal Dis 34: 13-25, 2019.

7. Paraskevopoulou MD and Hatzigeorgiou AG: Analyzing MiRNALncRNA interactions. Methods Mol Biol 1402: 271-286, 2016.

8. Kumar MM and Goyal R: LncRNA as a therapeutic target for angiogenesis. Curr Top Med Chem 17: 1750-1757, 2017.

9. Charles Richard JL and Eichhorn PJA: Platforms for investigating LncRNA functions. SLAS Technol 23: 493-506, 2018.

10. Qian X, Zhao J, Yeung PY, Zhang QC and Kwok CK: Revealing lncRNA structures and interactions by sequencing-based approaches. Trends Biochem Sci 44: 33-52, 2019.

11. Ma Y, Zhang J, Wen L and Lin A: Membrane-lipid associated lncRNA: A new regulator in cancer signaling. Cancer Lett 419: 27-29, 2018.

12. Wang HV and Chekanova JA: Long noncoding RNAs in plants. Adv Exp Med Biol 1008: 133-154, 2017.

13. Zhou H, Mangelsdorf M, Liu J, Zhu L and Wu JY: RNA-binding proteins in neurological diseases. Sci China Life Sci 57: 432-444, 2014.

14. Lujan DA, Ochoa JL and Hartley RS: Cold-inducible RNA binding protein in cancer and inflammation. Wiley Interdiscip Rev RNA 9: 10.1002/wrna.1462, 2018.

15. Köster T, Marondedze C, Meyer K and Staiger D: RNA-binding proteins revisited-the emerging arabidopsis mRNA interactome. Trends Plant Sci 22: 512-526, 2017.

16. Ferrè F, Colantoni $A$ and Helmer-Citterich M: Revealing protein-lncRNA interaction. Brief Bioinform 17: 106-116, 2016.

17. Panda AC, Martindale JL and Gorospe M: Affinity pulldown of biotinylated RNA for detection of protein-RNA complexes. Bio Protoc 6: e2062, 2016.

18. Cao L, Zhang P, Li J and Wu M: LAST, a c-Myc-inducible long noncoding RNA, cooperates with CNBP to promote CCND1 mRNA stability in human cells. Elife 6: e30433, 2017.

19. Gong C and Maquat LE: IncRNAs transactivate STAU1-mediated mRNA decay by duplexing with 3' UTRs via Alu elements. Nature 470: 284-288, 2011.

20. Su W, Wang L, Niu F, Zou L, Guo C, Wang Z, Yang X, Wu J, Lu Y, Zhang J, et al: LINC00857 knockdown inhibits cell proliferation and induces apoptosis via involving STAT3 and MET oncogenic proteins in esophageal adenocarcinoma. Aging (Albany NY) 11: 2812-2821, 2019.

21. Xia C, Zhang XY, Liu W, Ju M, Ju Y, Bu YZ, Wang W and Shao H: LINC00857 contributes to hepatocellular carcinoma malignancy via enhancing epithelial-mesenchymal transition. J Cell Biochem: Dec 3, 2018 (Epub ahead of print).

22. Dudek AM, van Kampen JG, Witjes JA, Kiemeney LA and Verhaegh GW: LINC00857 expression predicts and mediates the response to platinum-based chemotherapy in muscle-invasive bladder cancer. Cancer Med 7: 3342-3350, 2018.

23. Fléjou JF: WHO classification of digestive tumors: The fourth edition. Ann Pathol 31 (Suppl 5): S27-S31, 2011 (In French).
24. Gaspersz MP, Buettner S, van Vugt JL, de Jonge J, Polak WG, Doukas M, Ijzermans JNM, Koerkamp BG and Willemssen FE: Evaluation of the new American joint committee on cancer staging manual 8th edition for perihilar cholangiocarcinoma. J Gastrointest Surg 24: 1612-1618, 2020.

25. Livak KJ and Schmittgen TD: Analysis of relative gene expression data using real-time quantitative PCR and the 2(-Delta Delta C(T)) method. Methods 25: 402-408, 2001.

26. Carlevaro-Fita J and Johnson R: Global positioning system: Understanding long noncoding RNAs through subcellular localization. Mol Cell 73: 869-883, 2019.

27. Li Z, Chao TC, Chang KY, Lin N, Patil VS, Shimizu C, Head SR, Burns JC and Rana TM: The long noncoding RNA THRIL regulates TNF $\alpha$ expression through its interaction with hnRNPL. Proc Natl Acad Sci USA 111: 1002-1007, 2014.

28. Anders G, Mackowiak SD, Jens M, Maaskola J, Kuntzagk A, Rajewsky N, Landthaler M and Dieterich C: doRiNA: A database of RNA interactions in post-transcriptional regulation. Nucleic Acids Res 40: D180-D186, 2012.

29. Mura M, Hopkins TG, Michael T, Abd-Latip N, Weir J, Aboagye E, Mauri F, Jameson C, Sturge J, Gabra H, et al: LARP1 post-transcriptionally regulates $\mathrm{mTOR}$ and contributes to cancer progression. Oncogene 34: 5025-5036, 2015.

30. Galgano A, Forrer M, Jaskiewicz L, Kanitz A, Zavolan M and Gerber AP: Comparative analysis of mRNA targets for human PUF-family proteins suggests extensive interaction with the miRNA regulatory system. PLoS One 3: e3164, 2008.

31. Liu S, Li G, Li Q, Zhang Q, Zhuo L, Chen X, Zhai B, Sui X, Chen $\mathrm{K}$ and Xie T: The roles and mechanisms of YTH domain-containing proteins in cancer development and progression. Am J Cancer Res 10: 1068-1084, 2020.

32. Xiao W, Adhikari S, Dahal U, Chen YS, Hao YJ, Sun BF, Sun HY, Li A, Ping XL, Lai WY, et al: Nuclear m(6)A reader YTHDC1 regulates mRNA splicing. Mol Cell 61: 507-519, 2016.

33. Tanabe A, Tanikawa K, Tsunetomi M, Takai K, Ikeda H, Konno J, Torigoe T, Maeda H, Kutomi G, Okita K, et al: RNA helicase YTHDC2 promotes cancer metastasis via the enhancement of the efficiency by which HIF-1 $\alpha$ mRNA is translated. Cancer Lett 376: 34-42, 2016.

34. Tanabe A, Konno J, Tanikawa K and Sahara H: Transcriptional machinery of TNF- $\alpha$-inducible YTH domain containing 2 (YTHDC2) gene. Gene 535: 24-32, 2014.

35. Fanale D, Iovanna JL, Calvo EL, Berthezene P, Belleau P, Dagorn JC, Bronte G, Cicero G, Bazan V, Rolfo C, et al: Germline copy number variation in the YTHDC2 gene: Does it have a role in finding a novel potential molecular target involved in pancreatic adenocarcinoma susceptibility? Expert Opin Ther Targets 18: 841-850, 2014.

36. Kasowitz SD, Ma J, Anderson SJ, Leu NA, Xu Y, Gregory BD, Schultz RM and Wang PJ: Nuclear m6A reader YTHDC1 regulates alternative polyadenylation and splicing during mouse oocyte development. PLoS Genet 14: e1007412, 2018.

37. Liu X, Liu L, Dong Z, Li J, Yu Y, Chen X, Ren F, Cui G and Sun R: Expression patterns and prognostic value of $\mathrm{m}^{6} \mathrm{~A}$-related genes in colorectal cancer. Am J Transl Res 11: 3972-3991, 2019.

38. Popova VV, Kurshakova MM and Kopytova DV: Methods to study the RNA-protein interactions. Mol Biol (Mosk) 49: 472-481, 2015.

39. Sakata T, Ferdous G, Tsuruta T, Satoh T, Baba S, Muto T, Ueno A, Kanai Y, Endou H and Okayasu I: L-type amino-acid transporter 1 as a novel biomarker for high-grade malignancy in prostate cancer. Pathol Int 59: 7-18, 2009.

40. Kaira K, Oriuchi N, Imai H, Shimizu K, Yanagitani N, Sunaga N, Hisada T, Tanaka S, Ishizuka T, Kanai Y, et al: Prognostic significance of L-type amino acid transporter 1 expression in resectable stage I-III nonsmall cell lung cancer. Br J Cancer 98: 742-748, 2008.

41. Wang L, Cao L, Wen C, Li J, Yu G and Liu C: LncRNA LINC00857 regulates lung adenocarcinoma progression, apoptosis and glycolysis by targeting miR-1179/SPAG5 axis. Hum Cell 33: 195-204, 2020.

This work is licensed under a Creative Commons Attribution-NonCommercial-NoDerivatives 4.0 International (CC BY-NC-ND 4.0) License. 